\title{
Application Potential of Fuzzy and Regression in Optimization of MRR and Surface Roughness during Machining of C45 Steel
}

\author{
Santosh Madival ${ }^{1 *}$, Mohammed Riyaz Ahmed², Manjunath Lingappa Halappa1, \\ Lokesha Marulaiah ${ }^{3}$ \\ ${ }^{1}$ School of Mechanical Engineering, REVA University, Yelahanka, Bangalore-560064, India \\ 2 School of Electronics and Communication Engineering, REVA University, Yelahanka, Bangalore-560064, India \\ ${ }^{3}$ Department of Mechanical Engineering, Mangalore Institute of Technology and Engineering, Mangalore-574225, Moodbidri, India \\ *Corresponding author, e-mail: santoshmadival88@gmail.com
}

Received: 20 September 2018, Accepted: 08 January 2019, Published online: 18 March 2019

\begin{abstract}
In the machining industry, coolant has an important role due to their lubrication, cooling and chip removal functions. Using coolant can improve machining process efficiency, tool life, surface quality and it can reduce cutting forces and vibrations. However, health and environmental problems are encountered with the use of coolants. Hence, there has been a high demand for deep cryogenic treatment to reduce these harmful effects. For this purpose, $-196^{\circ} \mathrm{CLN}_{2}$ gas is used to improve machining performance. This study focuses on the prediction of surface roughness and material removal rate with cryogenically treated M2 HSS tool using fuzzy logic and regression model. The turning experiments are conducted according to Taguchi's L9 orthogonal array. Surface roughness and material removal rate during machining of C45 steel with HSS tool are measured. Cutting speed, feed rate, and depth of cut are considered as machining parameters. A model depended on a regression model is established and the results obtained from the regression model are compared with the results based on fuzzy logic and experiment. The effectiveness of regression models and fuzzy logic has been determined by analyzing the correlation coefficient and by comparing experimental results. Regression model gives closer values to experimentally measured values than fuzzy logic. It has been concluded that regression-based modeling can be used to predict the surface roughness successfully.
\end{abstract}

Keywords

surface roughness, material removal rate, regression, fuzzy logic

\section{Introduction}

In the manufacturing industry, machining process plays an important role. Generally, machining is defined as the removal of excess material from the workpiece in the form of chips. Using different methods, unwanted part can be removed without much difference in their results. The surface roughness $(R a)$ is an essential evaluation of the quality product since it considerably affects the staging of mechanical parts as well as the cost of production. Quality of surface (roughness) has a link with the mechanical properties such as corrosion resistance, fatigue behavior.

In small-scale industries, high-speed steel is in great use. HSS tool finds its application as a milling cutter, bits, drills, and broaches. When a too low cutting speed of bits is required, a manufacturer will think of carbide tools [1]. A standard procedure used in the ancient times to enhance the life of HSS cutting tools is by subjecting them to heat

treatment, which gives higher control beyond the scope of attributes viz. excellent wear resistance, hardness, and excellent toughness.

Cryogenic treatment is an additional subzero treatment (Vacuum) to the traditional heat treatment process, have been around for several years but certainly in its infancy when linked to heat-treating. Cryogenic treatment is the process of offering material to subzero temperatures (below $0{ }^{\circ} \mathrm{C}$ ) to increase the life of the tool, into morphological alterations that occur during treatment.

Various factors affect the quality surface roughness and material removal rate. The most prominent are cutting tool configurations and cutting parameters [2]. The influences of these parameters on the surface and material removal rate $(M R R)$ to be optimized by applying the Taguchi method. Hence, suitable prediction of surface 
roughness and material removal rate has been the focus of some researchers in the earlier decades [3].

Further, the Taguchi method applies an orthogonal array $(O A)$ design to study the effects of complete machining parameters by pilot experiments.

Employed the Taguchi method and regression analysis to evaluate machinability of AISI 4340 steel with afresh refined zirconia toughened alumina ceramic inserts. It has been observed that maximum contribution on tool wear is depth of cut [4]. The author analyzed the impact of deep cryogenic treatment on M2 HSS tool, later use in the automotive industry either shop floor or laboratory tests [5]. The result of each cutting parameter combination results in distinct tool life and surface roughness. Though, different parameters to control the varying levels for each one is intricate to establish the optimal combination that provides the maximum tool life and minimum surface roughness. The surface roughness evaluates the quality of the machined surface of the machined part, involving the most crucial condition characteristic. Few researchers assayed to prognosticate the surface quality of the machining process by mathematical algorithms.

The author investigated that the M2 HSS tool microstructure changes before and after cryogenic treatment [6]. It has found that treatment facilitates the formation of secondary carbon clustering and increase the carbide density in the following heat treatment process, thus increasing the wear rate of steel. Few authors studied the orthogonal turning process linked with the Taguchi method to select the best optimal controllable parameters, like as depth of cut, feed rate, and cutting speed [7]. The prime objective is to minimize the surface roughness, using Taguchi method analysis of the largest signal-to-noise ratio for surface roughness.

In this study, the authors analyzed the impact of individual cutting parameters on Material removal rate $(M R R)$ and Surface roughness $(R a)$. The primary aim is the identification of the optimal combination of turning parameters to achieve the good material removal rate and surface roughness. The results showed that fuzzy logic could be efficiently used to prognosticate MRR and surface roughness in the machining of C45 steel.

\section{Methods and Experimental procedure}

\subsection{Cryogenic Treatment method}

The $C T$ has conducted in a cryogenic chamber in which liquid nitrogen at $-196{ }^{\circ} \mathrm{C}$ is supplied through solenoid valve. The opening of the valve is controlled by a control panel to regulate the cooling cycle as per requirement. In this study, the controlled temperature in the chamber was $-185^{\circ} \mathrm{C}$ due to some practical constraints. Cryo-treatment setup where AISI M2 HSS tool samples are subjected to heat is shown in Fig. 1, two samples were submitted to the soaking period of $36 \mathrm{hrs}$ then followed by tempering at the rate of $150{ }^{\circ} \mathrm{C}$. The $C T$ cycle graphically shown in Fig. 2.

\subsection{Design of experiments}

The primary step for the design of experiments is to select the process parameters and classify the quality characteristics [8]. In cutting processes, the commonly used controlled parameters are the feed $(f)$, cutting speed $\left(V_{c}\right)$, and radial depth of cut ( $a p$ ). Apart from these parameters that could also be controlled by the geometry of the tool and insert radius. But these parameters acted as more regulated and linked with the tool properties; hence these are not the objective of this study. In the machining processes, the quality of surface roughness and material removal rate is most fundamental. The goal of this study is to find the optimal levels of machining parameters that maximize material removal rate and minimize the surface roughness [9].

The lathe manufacturer prescribed the experimental setup range for the machining parameters, i.e., the feed rate is $0.046-0.8 \mathrm{~mm} / \mathrm{rev}$, cutting speed is $30-1250 \mathrm{rpm}$, and the depth of cut in the range of $0.11-0.8 \mathrm{~mm}$. For this study, the three levels of the machining parameters selected are shown in Table 1.

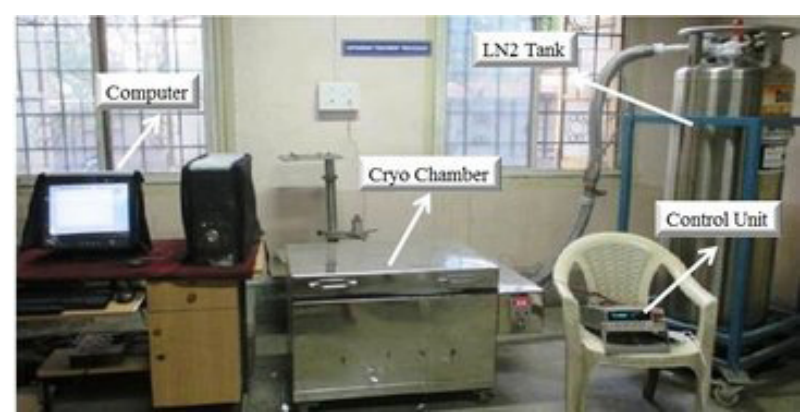

Fig. 1 A typical representation of cryogenic process

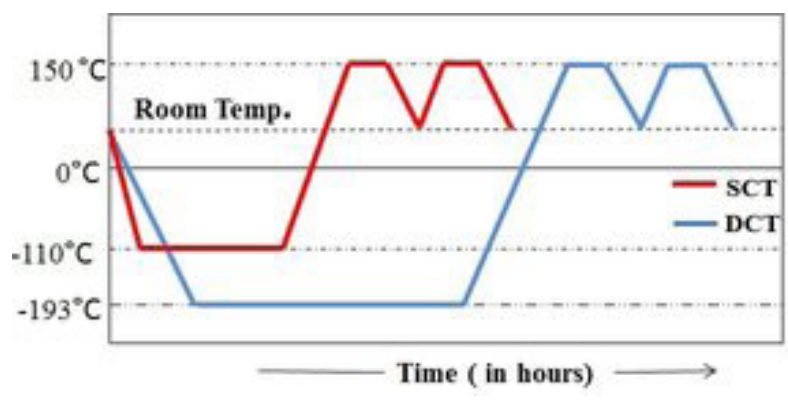

Fig. 2 Cryogenic cycle 
Table 1 Cutting parameters

\begin{tabular}{llccc}
\hline Symbol & Cutting parameter & Level 1 & Level 2 & Level 3 \\
\hline$A$ & Depth of cut $(\mathrm{mm})$ & 0.2 & 0.4 & 0.6 \\
$B$ & Cutting Speed $(\mathrm{m} / \mathrm{min})$ & 38.6 & 57.5 & 62.8 \\
$C$ & Feed Rate $(\mathrm{mm} / \mathrm{rev})$ & 0.046 & 0.062 & 0.087 \\
\hline
\end{tabular}

\subsubsection{Taguchi method}

Taguchi invented a unique design of orthogonal arrays $(O A)$ to analyze the cutting parameter with an $L_{9}$ pilot experiment is used [10]. To obtain experimental results were analyzed and then converted into a Signal-to-Noise $(S / N)$ Ratio. The use of $S / N$ ratio is to measure the quality characteristics differing or near to the desired values. Three classes of quality characteristics in the study of the $S / N$ ratio, i.e., smaller is the better, nominal the best, higher is the better [11].

\subsubsection{ANOVA}

A standard statistical technique ANOVA is used for defining the relationship between two or more groups of data or in other way degrees of difference based on the correlation of the mean value of a standard component [12]. In this work, Pareto ANOVA is used to evaluate the significance process parameter of the machining. It is a simplified method which is based on the Pareto principle. This method is easy and quick to investigate the response of the parametric design. This method does not require F-test. This method classifies the critical parameters and estimates the percentage impact of each parameter on varying quality characteristics. The application of both Pareto ANOVA method and SNR approach makes it less complex to investigate the results and therefore, execute it quickly to conclude.

\subsection{Modeling based on Fuzzy rule}

Fuzzy logic is a part of artificial intelligence; this method is the fundamental modeling technique. It is possible for the logic rule of a human being to be formed utilizing fuzzy logic especially in linguistic words the use of proper defining operations inputs and desired outputs. Moreover, currently it is estimated to be one of the extensively used powerful technologies due to its capacity for developing a fuzzy logic based intelligent system. However fuzzy logic is built on four fundamental theories such as linguistic variables, fuzzy sets, fuzzy IF-THEN rules, and possibility distributions, three are essential concepts in fuzzy, and most of the industrial applications use of fuzzy logic.

Fuzzy logic rules apply a fuzzy set approach which is employed to generate a fuzzy rule-based model. Generally, an establishment is done based on the rule, based on the usage of two classes of fuzzy logic rules such as TSK-type rules and Mamdani type rule.

In this work, the selection of input data is the depth of cut, feed rate, and cutting speed the response variable are surface roughness and material removal rate. In fuzzy logic, the primary stage is the selection of proper shapes of the membership function $\left(M_{f}\right)$ for generating the algorithm to select the cutting parameters. Representation of membership function is a graphical image of the magnitude of support of each machining parameter. It incorporates a weighting by each of the input value is processed, ultimately determines an output response.

A fuzzy rule for input value is as follows: cutting parameters cutting velocity, depth of cut, and the feed rates are classified into three membership functions such as Low, Medium, and High. Expressions for fuzzy output of surface roughness, and the material removal rate is divided into eight sets as Lowest, Lower, Low 1, Low 2, Medium, High 1, High 2, and Highest. The use of membership functions in fuzzy logic is an arbitrary curve. There are various types of membership functions available such as trapezoidal, triangular, Gaussian, etc. Surface roughness modelling in this work is carried using the triangular type of membership function.

Triangular type of membership function it needs three parameters; hence remaining functions needs more than three parameters. The simple formula is required, and their efficiency is best. Therefore, it is extensively used in a real-time application, and consequently, triangular type membership function is adopted in this paper. The triangular type membership function has three input parameters as follows $\{a, b, c\}$, where $\mathrm{a}, \mathrm{b}, \mathrm{c}$ refers to the fuzzy triangular triplet, and it defines the coordinates of $(x)$, three edges carrying membership function.

Triangle $(x ; a, b, c)=\left\{\begin{array}{ll}o, & x \leq a \\ \frac{x-a}{b-a}, & a \leq x \leq b \\ \frac{c-x}{c-b}, & b \leq x \leq c \\ o, & c \leq x\end{array}\right.$.

For example, cutting speed functions considered to be "Low", "Medium", and "High" as displayed in Fig. 3 (a). Similarly, for feed rate and depth of cut, the membership functions have fixed and are presented in Fig. 3 (b), (c). Output response shape function for surface roughness and material removal rate is shown in Fig. 4 (a), (b) respectively. 


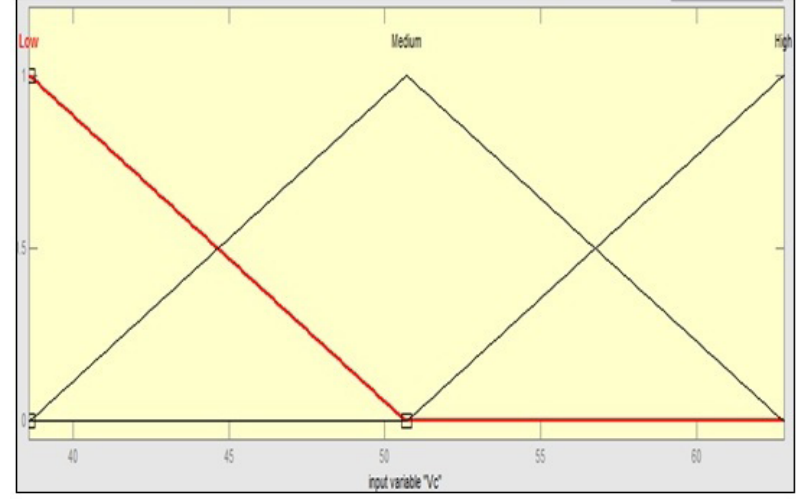

(a)

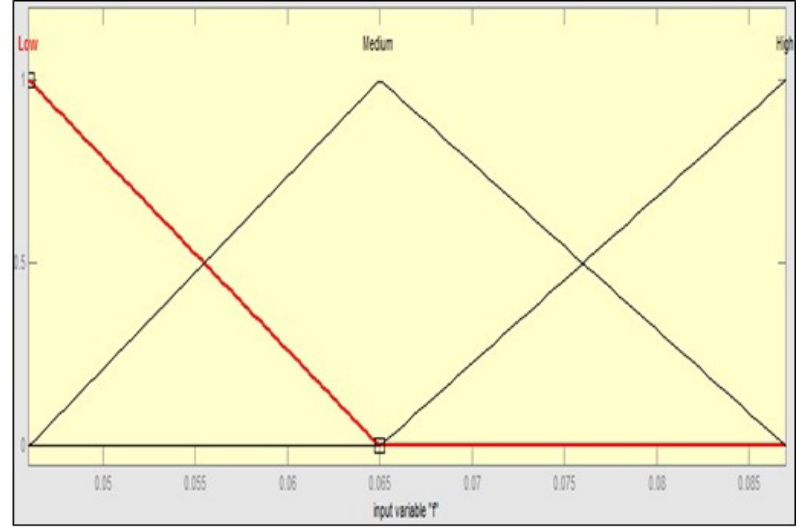

(b)

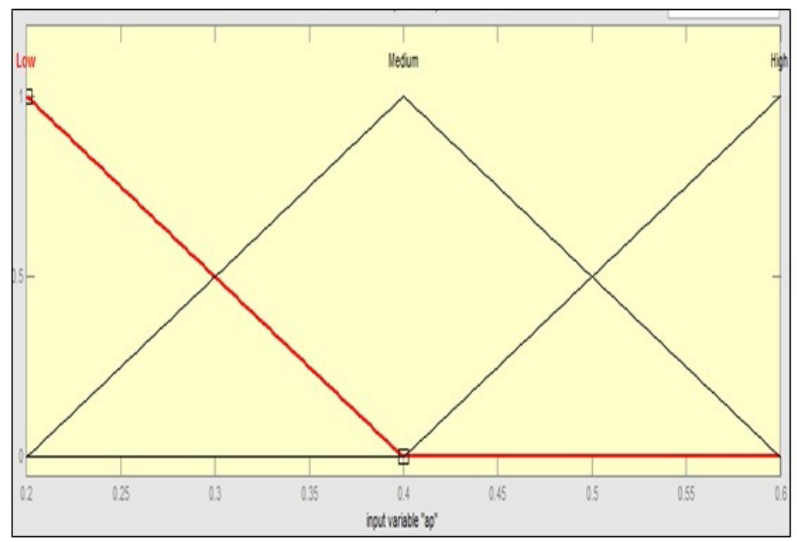

(c)

Fig. 3 Membership functions (a) $1^{\text {st }}$ input, (b) $2^{\text {nd }}$ input, (c) $3^{\text {rd }}$ input

A base of the fuzzy rule consists of a combination of "IF-THEN" a statement including three inputs parameters, $x_{1}, x_{2}$, and $x_{3}$ followed by two output responses $y_{1}$, $y_{2}$ respectively.

In this work, eight fuzzy rules were developed.

Rule 1 If " $V c$ " is low and " $f$ " is low, and " $a p "$ is low, and then "Ra" is $m f_{1}$.

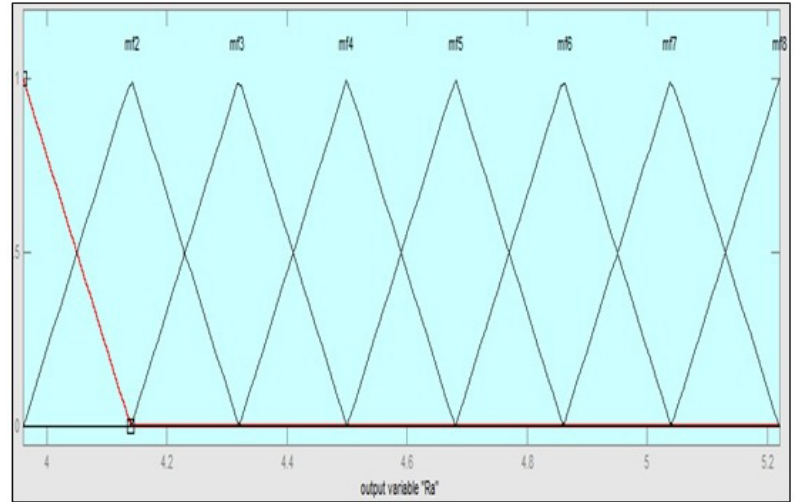

(a)

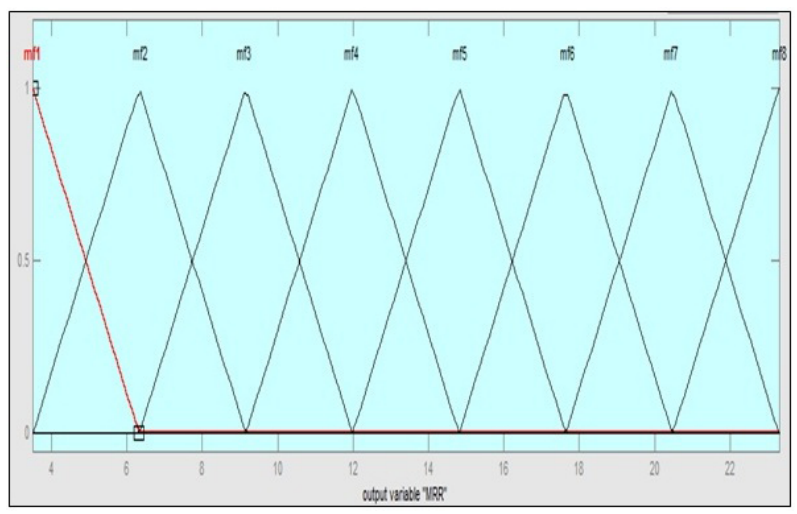

(b)

Fig. 4 Membership function (a) $R a$, output (b) $M R R$, output

Rule 2 If " $V c$ " is high and " $f$ " is low, and "ap" is high and then "Ra" is $m f_{2}$.

Rule 3 If " $V c$ " is high and " $f$ " is high, and "ap" is low and then "Ra" is $m f_{3}$.

Rule 4 If " $V c$ " is low and " $f$ " is high, and "ap" is high and then " $R a$ " is $m f_{4}$.

Rule 5 If " $V c$ " is high and " $f$ " is high, and "ap" is high and then "Ra" is $m f_{5}$.

Rule 6 If " $V c$ " is low and " $f$ " is low, and "ap" is high and then "Ra" is $m f_{6}$.

Rule 7 If " $V c$ " is low and " $f$ " is high, and "ap" is low and then "Ra" is $m f_{7}$.

Rule 8 If " $V c$ " is high and " $f$ " is low, and "ap" is low and then "Ra" is $m f_{8}$.

Based on the experimental results, the above rules developed as shown in Table 2. The pilot experiment was conducted through a turning operation of cylindrical workpiece C45 steel by Untreated, and Cryo-treated M2 HSS tool. The initial diameter of the workpiece is $32 \mathrm{~mm}$ and length $300 \mathrm{~mm}$. Experimental condition as shown in Table 3. 
Table $2 \mathrm{~L}_{9}$ Orthogonal array experimental runs

\begin{tabular}{cccccc}
\hline$A$ & $B$ & $C$ & $\begin{array}{c}R a(U T) \\
\mu \mathrm{m}\end{array}$ & $\begin{array}{c}R a(C T) \\
\mu \mathrm{m}\end{array}$ & $\begin{array}{c}M R R \\
\mathrm{~cm}^{3} / \mathrm{m}\end{array}$ \\
\hline 0.2 & 38.6 & 0.046 & 3.40 & 3.45 & 3.5 \\
0.2 & 57.5 & 0.062 & 3.94 & 3.69 & 7.1 \\
0.2 & 62.8 & 0.087 & 4.21 & 3.93 & 10.9 \\
0.4 & 38.6 & 0.062 & 3.60 & 3.43 & 9.5 \\
0.4 & 57.5 & 0.087 & 4.03 & 3.69 & 19.9 \\
0.4 & 62.8 & 0.046 & 4.15 & 3.71 & 11.5 \\
0.6 & 38.6 & 0.087 & 3.58 & 3.40 & 20.0 \\
0.6 & 57.5 & 0.046 & 3.84 & 3.52 & 15.8 \\
0.6 & 62.8 & 0.062 & 4.02 & 3.67 & 23.3 \\
\hline
\end{tabular}

Table 3 Experiment conditions

\begin{tabular}{ll}
\hline Machine tool: & Lathe Machine, 4 HP \\
\hline Work Specimen & Hot rolled C45 steel \\
Material: & M2 HSS \\
Cutting tool: & Kistler dynamometer \\
Tool holder: & \\
Tool Signature & $60^{\circ}$ \\
Back rake angle: & $60^{\circ}$ \\
Side rake angle: & $60^{\circ}$ \\
End relief angle: & $30^{\circ}$ \\
End cutting edge angle: & $15^{\circ}$ \\
Side cutting edge angle: & 0.4 mm \\
Nose radius: & \\
Process Parameters & $38.6,57.5, \& 62.8(\mathrm{~m} / \mathrm{min})$ \\
Cutting velocity: & $0.046,0.062, \& 0.087(\mathrm{~mm} / \mathrm{rev})$ \\
Feed rate: & $0.2,0.4, \& 0.6(\mathrm{~mm})$ \\
Depth of cut: & Dry machining \\
Environment: &
\end{tabular}

\section{Result and Discussion}

The response of machining operations scrutinized by employing the ANOVA and $S / N$ ratio. The result analyses, best optimal machining parameters for maximum material removal rate and minimum surface roughness are obtained and verified.

\section{1 (ANOVA) Analysis}

The design of ANOVA was to examine which parameters significantly influenced the performance properties. Table 4 and Table 5 depict the results of ANOVA for the surface roughness of untreated and cryo-treated respectively. Table 6 shows the ANOVA results of MRR; It found that the adjustment of machining parameters given in Table 1 .
The effects of ANOVA shows that the cutting speed is the most vital on both untreated and treated machining process parameters for affecting on the surface roughness $(R a)$. In the case of $M R R$, the depth of cut is a most affecting parameter shown in Table 6; surface roughness features due to its most massive percentage contribution cutting speed $(89.22 \% U T)$ and $(72.15 \% C T)$ among the selected process parameters shown in Table 4, 5. Table 6 further indicates that the percentage contribution of $M R R$ is $66.91 \%$ depth of cut. In this experimental outcome for surface roughness, material removal rate depicted in Tables 4-6 respectively.

Regression analysis was carried out modeling and analyzing of several variables which are the relationship between dependent variables and one or more independent variables. Mathematical models based on the depth of cut, cutting speed, and feed rate are obtained from regression analysis to predict surface roughness and material removal rate.

Table 4 Analysis of variance $R a(U T)$

\begin{tabular}{lccccc}
\hline Source & DF & Seq SS & Adj MS & $P$-value & PC $(\%)$ \\
\hline Regression & 3 & 0.591 & 0.197 & 0.001 & 94.54 \\
$A$ & 1 & 0.002 & 0.002 & 0.610 & 32.00 \\
$B$ & 1 & 0.557 & 0.557 & 0.000 & 89.22 \\
$C$ & 1 & 0.034 & 0.031 & 0.085 & 15.00 \\
Error & 5 & 0.034 & 0.006 & & 5.46 \\
Total & 8 & 0.062 & & & 100 \\
\hline$R^{2}=94.54 \%, R^{2}(a d j)=91.27 \%$ & & &
\end{tabular}

Table 5 Analysis of variance $R a(C T)$

\begin{tabular}{lccccc}
\hline Source & DF & Seq SS & Adj MS & $P$-value & PC (\%) \\
\hline Regression & 3 & 0.230 & 0.076 & 0.000 & 96.56 \\
$A$ & 1 & 0.038 & 0.038 & 0.005 & 16.07 \\
$B$ & 1 & 0.172 & 0.172 & 0.000 & 72.22 \\
$C$ & 1 & 0.019 & 0.019 & 0.018 & 8.00 \\
Error & 5 & 0.008 & 0.001 & & 3.44 \\
Total & 8 & 0.239 & & & 100 \\
\hline
\end{tabular}

$R^{2}=96.56 \%, R^{2}(a d j)=94.50 \%$

Table 6 Analysis of Variance $M R R$

\begin{tabular}{lccccc}
\hline Source & DF & Seq SS & Adj MS & $P$-value & PC (\%) \\
\hline Regression & 3 & 331.63 & 110.54 & 0.002 & 94.14 \\
$A$ & 1 & 235.63 & 235.62 & 0.001 & 66.89 \\
$B$ & 1 & 29.52 & 29.524 & 0.044 & 8.38 \\
$C$ & 1 & 66.48 & 66.480 & 0.010 & 18.87 \\
Error & 5 & 20.63 & 4.126 & & 5.86 \\
Total & 8 & 352.26 & & & 100 \\
\hline$R^{2}=94.14 \%, R^{2}(a d j)$ & & & & &
\end{tabular}

$R^{2}=94.14 \%, R^{2}($ adj $)=90.63 \%$ 
The mathematical models for surface roughness were as follows:

For surface roughness $(R a, U T)$

$R_{a}=2.403-0.092 A+0.02397 B+3.49 C$.

For $(R a, C T)$

$R_{a}(C T)=2.8828-0.4000 A+0.01333 B+2.791 C$.

For material removal rate $(M R R)$

$$
M R R=-18.74+31.33 A+0.1744 B+161.1 C .
$$

For multiple linear regression analysis $(M R A), R$-value indicates the coefficient of correlation and it should lie between the 0.8 and 1 . In this investigation, the surface roughness (UT, CT) and $M R R$ values of $R^{2}$ lie between 0.94 and 0.96 which are close to 1 . So, the patterns are quite adequate to predict surface roughness and material removal rate.

To estimate the efficacy of the designed fuzzy model, the experimental and regression values were matched with the predicted results. The correlations among the experimental and predicted values are analyzed, the plots for experimental values and predicted fuzzy values are alike and the differences among experimental and predicted fuzzy values were minimum.

The absolute error for $U T$ and $C T$ was observed to be smaller than $9 \%$ and $6 \%$ respectively for both $R a$ and $M R R$. From the analysis of results, it can be resolved that fuzzy modeling can be used successfully for predicting the $R a$ and $M R R$ in the turning of $\mathrm{C} 45$ medium carbon steel. For scatter plots using linear regression for the experimental values and predicted values are depicted in Fig. 5, the performance of the evolved fuzzy logic model was found by $R$-value (coefficient of determination). In the fuzzy model, $R^{2}$ values were found that $99.84 \%$ for $R a(U T)$ and $95.41 \%$ for $R a(C T)$ was used, which confirmed that the high correlation existed among the fuzzy predicted and experimental values. In regression, $R$-value was found to be $99.71 \%$.

The above results of surface roughness and $M R R$ models were designed using fuzzy logic modeling and models were tested that experimental results fitted with predicted results when $R$ values were the consideration into account. To validate the developed model, tests need to be carried out.

The designed models can be validated by confirmation experiments $(C E)$ and the findings of the confirmation experiment are summarized as shown in Fig. 7 (a), (b). The two confirmation experiments were carried out at the cutting speed of $62.8 \mathrm{~m} / \mathrm{min}$, a feed rate of $0.087 \mathrm{~mm} / \mathrm{rev}$ and depth of cut $0.6 \mathrm{~mm}$, which were the heaviest cutting

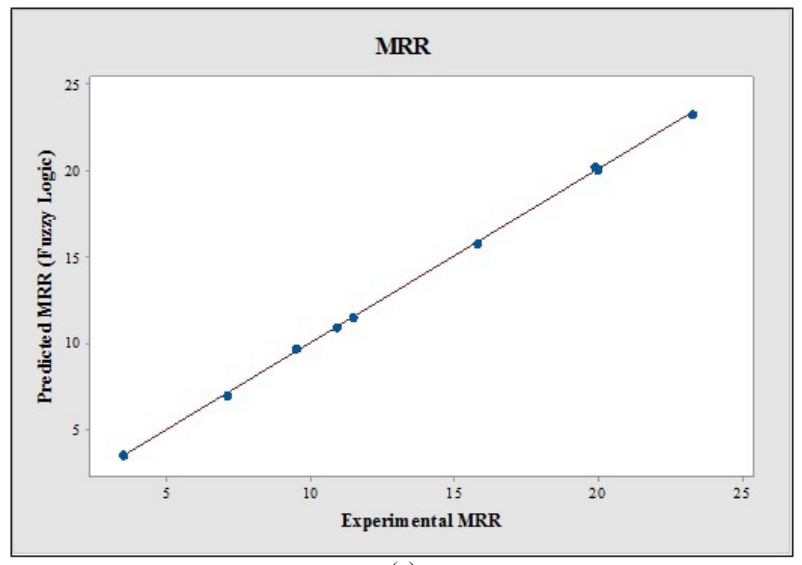

(a)

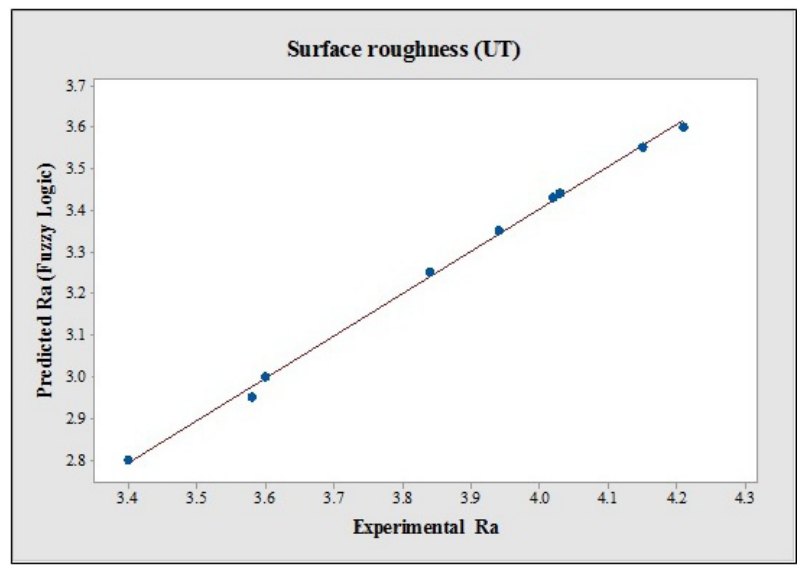

(b)

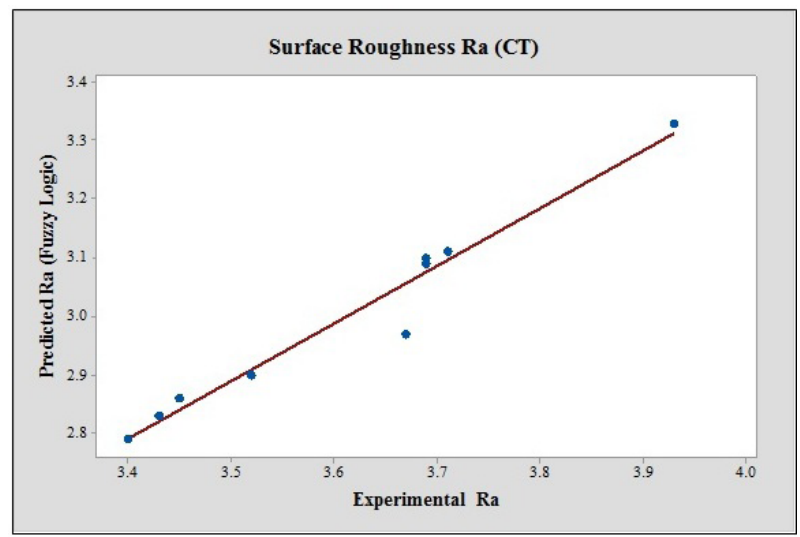

(c)

Fig. 5 Scatter plot for experimental $R a$ values and their fuzzy predicted values for $U T$ (a), for $C T(\mathrm{~b})$, experimental $M R R$ values and their fuzzy predicted values (c).

conditions investigated in this study. The comparison of experimental results and predicted values are also shown in Fig. 6 (a)-(c). From these results, it can be concluded that a regression model gives more accurate results than the fuzzy logic model for predicting both the surface roughness and $M R R$. 


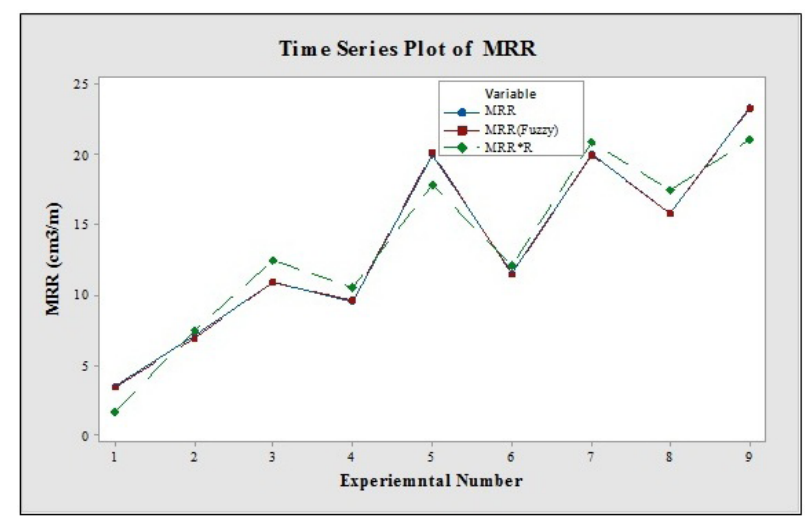

(a)

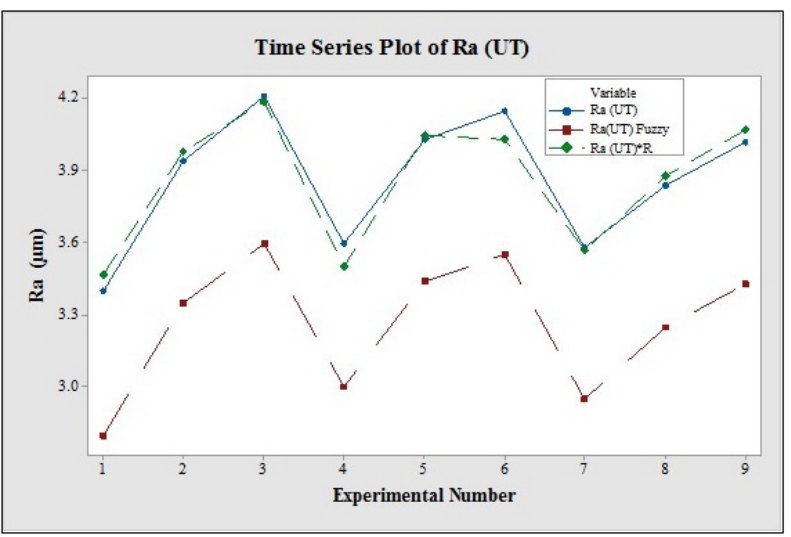

(b)

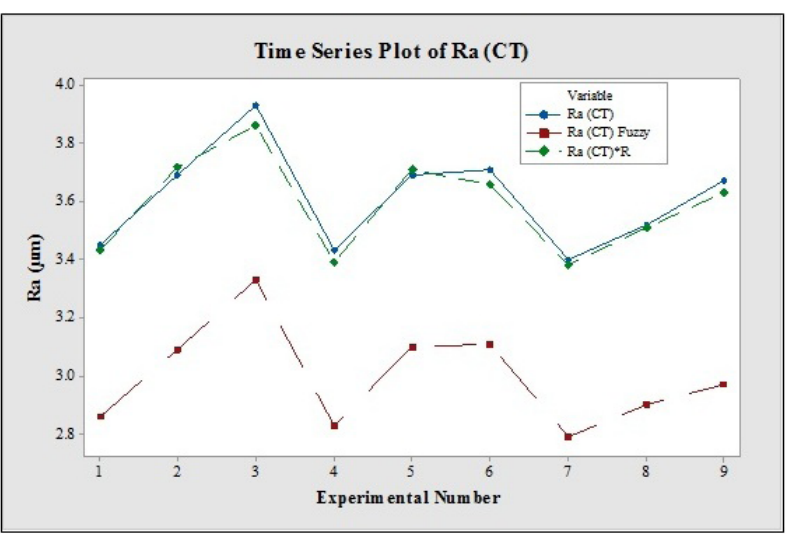

(c)

Fig. 6 Difference between experimental runs and predicted results for $R a(U T)$ (a), $R a(C T)$ (b), and for $M R R$ (c)

The error can be calculated as follows:

Error $=\frac{\text { Predicted values }- \text { Measured values } * 100}{\text { Measured values }}$.

The absolute maximum error for $M R R$ was observed to be $52.83 \%$ when the regression model was used while for fuzzy logic model gave $1.14 \%$ error. For $R a(U T)$ this fuzzy logic model gave $17.64 \%$ error, while for the regression model error was $2.05 \%$. For $R a(C T)$ the regression

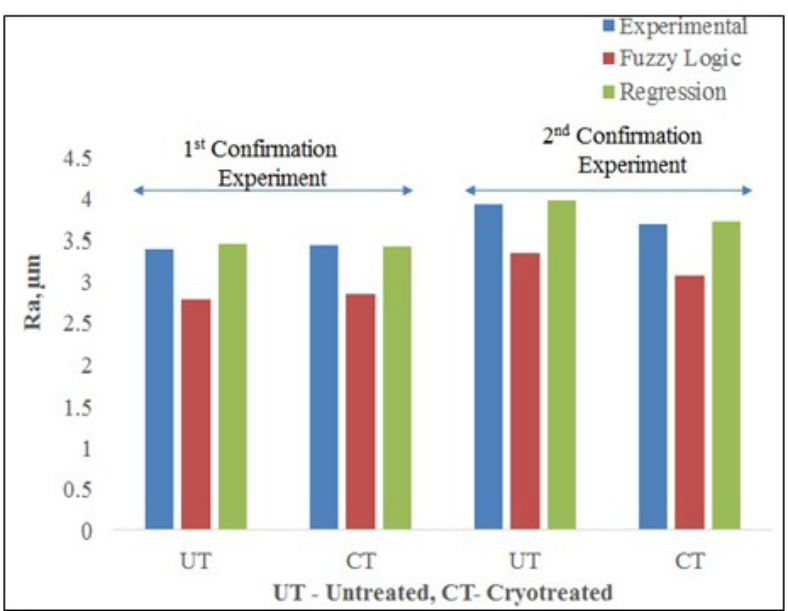

(a)

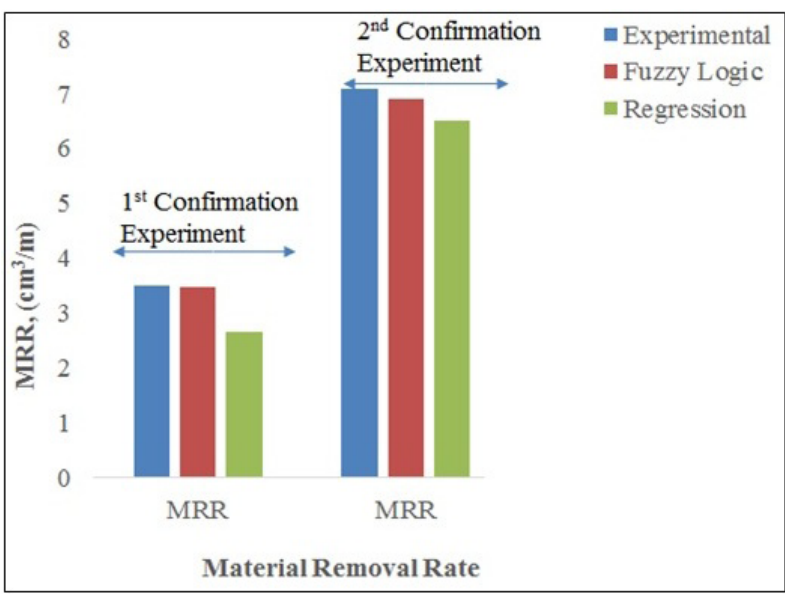

(b)

Fig. 7 (a) Results of confirmation experiment for $R a(U T, C T)$

(b) Results of confirmation experiments for $M R R$

model gave $0.5 \%$ error, for fuzzy logic model error was $17.10 \%$.

In this investigation, cutting speed, feed rate and depth of cut were utilized as the machining factors during turning of C45 medium carbon steel and models for surface roughness and material removal rate was developed. Results exhibited that the regression model was better than a fuzzy logic model for prognosticating the surface roughness ( $U T, C T)$. In the case of $M R R$ fuzzy logic shows less error compared with the regression model.

\section{Conclusions}

In this research, the model depends on the regression model approach on the forecast of machining parameters and the results obtained from the regression model was correlated with the results based on experiment and fuzzy logic. In general, cryogenically treated tool gave less surface roughness values compared with an untreated tool. 
From the result analysis, it was decided that fuzzy logic modeling gave feasible prognostications, the $R^{2}$ values of the linear regression modeling can successfully be used for predicting the surface roughness, in the turning of $\mathrm{C} 45$

\section{References}

[1] Silvio, T. "Animation: The New Performance?", Journal of Linguistic Anthropology, 20(2), pp. 422-438, 2010. https://doi.org/10.1111/j.1548-1395.2010.01078.x

[2] O'Brien, S. J., Johnson, W. E. "Big Cat Genomics", Annual Review of Genomics and Human Genetics, 6(1), pp. 407-429, 2005. https://doi.org/10.1146/annurev.genom.6.080604.162151

[3] Lee, Y., Terzopoulos, D., Walters, K. "Realistic Modeling for Facial Animation", In: 22 ${ }^{\text {nd }}$ Annual Conference on Computer Graphics and Interactive Techniques, New York, USA, 1995, pp. 55-62. https://doi.org/10.1145/218380.218407

[4] Muyan-Özçelik, P., Glavtchev, V., Ota, J. M., Owens, J. D. "Chapter 32 - Real-Time Speed-Limit-Sign Recognition on an Embedded System Using a GPU", In: GPU Computing Gems Emerald Edition, Application of GPU Computing Series, 3rd ed., Elsevier Inc., Burlington, USA, 2010, pp. 497-515. https://doi.org/10.1016/B978-0-12-384988-5.00032-2

[5] Bridson, R., Fedkiw, R., Anderson, J. "Robust treatment of collisions, contact and friction for cloth animation", Transactions on Graphics (TOG), 21(3), pp. 594-603, 2002.

https://doi.org/10.1145/566654.566623

[6] Molinari, A., Pellizarri, M., Gialanella, S. "Effect of deep cryogenic treatment on the mechanical properties of tool steels", Journal of Materials Processing Technology, 118(1-3), pp. 350-355, 2001. https://doi.org/10.1016/S0924-0136(01)00973-6

[7] Selvaraj, D. P., Chandramohan, P. "Optimization of Surface Roughness of AISI 304 Austenitic Stainless Steel in Dry Turning Operation Using Taguchi Design Method", Journal of Engineering Science and Technology, 5(3), pp. 293-301, 2010. steel. Confirmation experiments exhibited that the regression model was better than the fuzzy logic for predicting the surface roughness. While for $M R R$ fuzzy logic model exhibits better than the regression model.

[8] Yu, L. X. "Pharmaceutical Quality by Design: Product and Process Development, Understanding, and Control", Pharmaceutical Research, 25(4), pp. 781-791, 2008. https://doi.org/10.1007/s11095-007-9511-1

[9] Tosun, N., Cogun, C., Tosun, G. "A study on kerf and material removal rate in wire electrical discharge machining based on Taguchi method", Journal of Materials Processing Technology, 152(3), pp. 316-322, 2004.

https://doi.org/10.1016/j.jmatprotec.2004.04.373

[10] Dubey, A. K., Yadava, V. "Robust parameter design and multi-objective optimization of laser beam cutting for aluminium alloy sheet", The International Journal of Advanced Manufacturing Technology, 38(3-4), pp. 268-277, 2008. https://doi.org/10.1007/s00170-007-1105-X

[11] Santosh, Manjunatha, L. H., Lokesha, M.,Ajaykumar, B. S. "A review: Mechanical Properties of HSS Steel by deep CryoTreatment", IOP Conference Series: Materials Science and Engineering, 376, article ID: 012098, 2018. https://doi.org/10.1088/1757-899X/376/1/012098

[12] Mertler, C. A., Reinhart, R. V. "Advanced and Multivariate Statistical Methods: Practical Application and Interpretation", 4th ed., Routledge, Phoenix, USA, 2016. https://doi.org/10.4324/9781315266978 